\title{
Cultural and Morphological Characterization of Rhizoctonia solani f. sp. sasakii Isolates Collected from Different Districts of Andhra Pradesh
}

\author{
Bindu Madhavi Gopireddy ${ }^{1 *}$, G. Uma Devi ${ }^{2}$, K.Vijayakrishna Kumar ${ }^{1}$, \\ T. Ramesh Babu ${ }^{1}$ and T.C.M.Naidu ${ }^{1}$ \\ ${ }^{1}$ Acharya N G Ranga Agricultural University, Guntur, Andhra Pradesh, India \\ ${ }^{2}$ Professor Jayasankar Telangana State Agricultural University, Hyderabad, Telangana, India \\ *Corresponding author
}

\section{A B S T R A C T}

\begin{tabular}{|l|}
\hline Ke y w o r d s \\
Maize, BLSB, \\
Rhizoctonia solani f. \\
sp. sasakii, \\
$\begin{array}{l}\text { Morphological, } \\
\text { Cultural } \\
\text { characterization. }\end{array}$ \\
\hline Article Info \\
\hline $\begin{array}{l}\text { Accepted: } \\
\text { 26 September 2017 } \\
\text { Available Online: } \\
\text { 10 November 2017 }\end{array}$ \\
\hline
\end{tabular}

Sixty BLSB affected maize samples were collected from three districts and pathogen was isolated and identified based on morphological, cultural and sclerotial characters using standard descriptions of IMI. Light microscopic studies revealed that all the isolates of $R$. solani f. sp. sasakiiare characteristically branched out at right angle in the distal end of the cell and showed a characteristic constriction at the point of branching. Formation of septum near the point of origin of the branch / adjacent to branch was present in most of the isolates except for isolates RS 44, RS 48, RS 58 and RS 59, where in the septum was slightly away from the origin of branching. The hyphal width of all the 60 isolates varied from $5.05 \mu \mathrm{m}$ (RS 52) to $7.98 \mu \mathrm{m}$ (RS 15). Out of 60 maize isolates, eight isolates i.e., RS 7, RS 8, RS 9, RS 10, RS 11, RS 12 RS 16 and RS 26 produced barrel shaped monilliod cells and the remaining isolates produced irregular shaped monilliod cells. Clamp connections were absent in all the isolates, multinucleate and the number of nuclei per cell varied from five to seven in the isolates.

\section{Introduction}

Maize (Zea mays L.) is one of the most versatile emerging crops having wider adaptability under varied agro-climatic conditions. Globally, maize is known as queen of cereals due to its high genetic yield potential. Among the potential factors that limit maize production, fungal diseases are reported to cause extensive crop yield reduction in many countries and are considered as a priority in disease management practice (Agrios, 2005). Of different fungal diseases affecting maize cultivation, banded leaf and sheath blight (BLSB) incited by Rhizoctonia solani f. sp. sasakii Exner (Thanatephorus sasakii (Shirai)
$\mathrm{Tu}$ and Kimbrough) ( $\mathrm{Tu}$ and Kimbrrough, 1978 ) is an economically significant disease causing huge losses in all crop growing areas of the world. Increased incidence of BLSB has been observed in rice fallow maize crop (zero tillage) in different districts of Andhra Pradesh. Effective management of BLSB in maize is possible only when the pathogen is eliminated completely or the propagules are brought down below economic threshold limits at field level. Control measures used were partly effective because $R$. soiani is able to produce sclerotia that can persist in the soil for at least two years $(\mathrm{Ou}, 1985)$. The pace of 
development and durability of resistant varieties had been slow and unreliable despite tremendous advancements in the field of plant genetic engineering. Variability of the pathogen plays major role in resistance breeding hence the cultural and morphological characterization of the isolates was studied.

\section{Materials and Methods}

To study the morphology of hyphae of each BLSB pathogenic isolate, four day old-fungal hyphae grown on PDA medium was taken and stained with 0.1 per cent lactophenol cotton blue on microscopic slides for recording, angle of branching, septation, presence of monilioid cells, presence of clamp connections, type of septum and hyphal width and number of nuclei using Olympus CX31 microscope with ProgRes. CT3 image analyser.

\section{Cultural characteristics of Rhizoctonia solani f. sp. sasakii isolates (Fig. 1)}

\section{Growth of fungal pathogen}

The isolates were grown on PDA medium in Petri dishes at $27+2^{\circ} \mathrm{C}$ until the hyphae reached the periphery of the petridishes for determination of color, abundance of mycelium, zonation and sclerotial characters.

\section{Colony characters}

Abundance of mycelium was compared with the key given by Burpee et al., (1980).

The abundance was characterized into the following four categories.

Slight: Aerial mycelium does not obscure surface mycelium
Moderate: Aerial mycelium obscure surface mycelium and does not touch the cover of Petri dishes

Abundant: Aerial mycelium obscure surface mycelium and touches the cover of Petri dishes

No aerial mycelium

Colony color was determined with the help Munsell's soil colour chart (Munsell color Company, Inc., 1954).

The culture and key color cards were placed side by side against white background under sunlight for comparison (Burpee et al., 1980).

Observations for colony color were recorded 10 days after incubation.

Based on the colony pigmentation, the cultures were assigned to different groups based on dominant spectral color.

\section{Sclerotial characteristics of Rhizoctonia solani f. sp. sasakii isolates}

Sclerotial characteristics were also compared with the key given by Burpee et al., (1980).

\section{Location of sclerotia}

Based on location of sclerotial production in the culture the isolates of $R$. solani $\mathrm{f}$. $\mathrm{sp}$. sasakii were categorized into following groups

Aerial: Sclerotia formed with in aerial mycelium

Embedded: Sclerotia formed with in substrate

\section{Size of sclerotia}

The size of sclerotia of the sixty isolates that were 10-day-old were observed by keeping 
the Petri dishes under the stereo binocular microscope and were classified as (a) Large (b) Small

\section{Colour of the sclerotia}

It was categorized in to 4 groups a) Light brown (b) Brown (c) Dark brown (d) Deep dark brown

\section{Location and Pattern of sclerotial formation}

Location of sclerotia produced by different isolates in the Petri dishes containing PDA were observed and recorded as sclerotia produced on the surface of agar (aerial) or submerged in the medium. The pattern of the sclerotial production was also studied and the isolates were divided into different categories based on their distribution on the culture. The production of sclerotia by different isolates was recorded as more or less circular manner concentrated towards periphery; irregularly scattered but more towards the centre of the colony; irregular very sparsely scattered and scattered irregularly all over the colony surface. Sclerotial types were mainly divided into two categories as follows.

Sclerotia with rough border

Sclerotia with smooth border

\section{Results and Discussion}

Morphological variability among the isolates of Rhizoctonia solani f. sp. sasakii of maize

Morphological characters are the important basic factors for identification of a fungus and its variability. Studies on morphological characteristics of $R$. solani $\mathrm{f}$. sp. sasakii maize isolates (60 numbers) and RS 61 were studied and the results are presented in Table 1.

Light microscopy studies revealed that all the isolates of $R$. solani f. $\quad$ sp. sasakii characteristically branched out at right angle in the distal end of the cell (Plate 1).

\section{Constriction at the point of branching}

All isolates showed a characteristic constriction at the point of branching. Formation of septum near the point of origin of the branch / adjacent to branch was present in most of the isolates while in isolates RS 44, RS 48, RS 58, RS 59 the septum was slightly away from the origin of branching.

\section{Hyphal width}

The hyphal width of all the 60 isolates varied from $5.05 \mu \mathrm{m}$ (RS 52 from Krishna district) to $7.98 \mu \mathrm{m}$ (RS 15 from Prakasam district). The hyphal widths of most of the isolates were at par with each other. However, the differences in hyphal width observed among the other isolates were non- significant.

\section{Monilioid cells}

In addition to ordinary vegetative hyphae, $R$. solaniproduces simple or branched chains of short broad cells, which may be hyaline or brown, barrel shaped, pyriform, irregular, or lobate known as monilioid cell (Plate1). Out of 60 maize isolates, eight isolates i.e., RS 7, RS 8, RS 9, RS 10, RS 11, RS 12 RS 16 and RS 26 produced barrel shaped monilioid cells. The remaining isolates produced irregular shaped monilioidcells .

Clamp connections: were absent in all the isolates

Observation for the mycelium branching out at right angles, presence of characteristic constriction at the point of branching and formation of septum near the point of origin of the branch, hyphal width $>5.00 \mu \mathrm{m}$, presence of monilioid cells, absence of clamp connections were the characters of immense 
taxonomical importance which were described by the previous workers Duggar (1915), Matsumato (1921), Thomas (1925).

Number of nuclei

All the isolates under present investigation were found to be multinucleate and the number of nuclei per cell varied from five to seven. Isolates RS 21, RS 23, RS 25, RS 26, RS 27 had statistically maximum number (7) of nuclei per cell (Table 1).

The classification of Rhizoctonia solanihas been done on the basis of hyphal and cultural, morphology, nuclear condition, hyphal anastomosis and morphology of teleomorphs. The present findings on morphological variability among $R$. solani isolates are in accordance with Sneh et al., (1991), Amita Singh et al., (1999), Meena et al., (2001) and Srinivas (2002) in maize, Singh et al., (2002) and Basu et al., (2004) in rice.

\section{Cultural variability of $R$. solani isolates}

\section{Colony colour}

The colour of the colony varied from white to dark brown. Based on pigmentation dominant spectral colour from Munsell's soil colour chart (1954), the cultures were assigned to five colour groups with respective shade numbers i.e, Group I- white, Group IIyellowish white, Group III-pale brown and Group IV brown and Group V dark brown (Table 1).

Among the 60 isolates studied, 11 isolates belonged to group I, 9 isolates with yellowish white were assigned in group II, 22 isolates in Group III, ten isolates in group IV and eight in Group V. The variation in the colour of the colony might be attributed to the production of pigments by the pathogen. The differences in the intensity of the colour might also correspond to the amount of pigments released by respective isolate in the media. The colour production may also be due to release of other secondary metabolites like toxins. Amita Singh et al., (1999) assigned Munsell'ssoil colour chart shade number to the colony colour of $R$. solani isolates from rice, maize, soybean, mung beans and cotton. Further, Akhtar et al., (2009) stated that the colony colour of $R$. solani maize isolates $H c$ and $I t$ were brown whereas the isolates $B c, J r$ and $R f$ had white colour. Studies on cultural characteristics revealed that the colony colour of different $R$. solani isolates varied from white to brown on PDA (Khodayari et al., 2009). The results are also in agreement with the observations of other researchers (Sneh et al., 1991; Sweetingham and Mac Nish, 1994; Amita Singh et al., 1999). Srinivas (2002) categorised maize isolates of $R$. solanicausing BLSB disease based on colony pigmentation.

\section{Abundance of mycelium}

Among 60 isolates, 27 isolates produced abundant mycelium, while 18 isolates have moderate mycelium and the remaining 15 isolates recorded slight/ sparse mycelium.

\section{Colony diameter and growth rate}

The data presented in Table 1 on colony diameter and growth rate revealed that there were significant differences among the isolates after 72 hours of incubation on PDA medium. Among the 60 isolates, 29 isolates recorded as fast growers (more than $40 \mathrm{~mm}$ growth) and 21 as moderate (35-40mm growth) and ten recorded slow growth (30-35 $\mathrm{mm}$ ) after $72 \mathrm{~h}$ of incubation.

The cultural characteristics studied among the $R$. solani isolates with respect to the colony colour, abundance of mycelium, colony diameter and growth rate revealed the existence of significant variation. 
Distinct differences were observed in the colony appearance and the isolates were categorised into different groups based on texture and abundance of mycelium. The difference in the colony growth was distinct in 27 isolates. These isolates produced abundant aerial cottony mycelial growth which may be due to the inherent nature of these isolates to go for quick and profuse mycelial growth in early stages of growth before setting the sclerotia.

Similar observations have been made by Toda et al., (1999) who divided Rhizoctonia AG-D isolates into two subgroups AG-D (I) and AG-D (II), based on the results of cultural characteristics; Srinivas (2002) categorised the $R$. solani f. sp. sasakii isolates from maize based on texture and abundance of their mycelia growth and colony appearance. Similarly Guleria et al., (2007) used cultural characters for differentiating the $R$. solani isolates from rice.

Significant variations with respect to colony growth and growth rate were also recorded among the isolates under the study. The isolates RS 34, RS 26, RS 25 having fast growth rate were found more virulent as they induced susceptible reaction on maize. Meena et al., (2003) observed that the fast growing isolate of $R$. solani from maize was found to be more virulent on a susceptible maize cultivar.

Similarly, Guleria et al., (2007), Thind and Aggarwal (2008) and Khodaryari et al., (2009) stated that the $R$. solani isolates from rice were fast growing with $>20 \mathrm{~mm}$ mycelia growth rate per day indicating their fast growing nature. Rapid growth rate among $R$. solani isolates have also been reported by Peltier (1916), Matz (1921), Matsumoto (1934) and Parmeter and Whitney (1970).

Fig.1 Cultural and sclerotial characteristics of different isolates of Rhizoctonia solani f. sp. sasakii

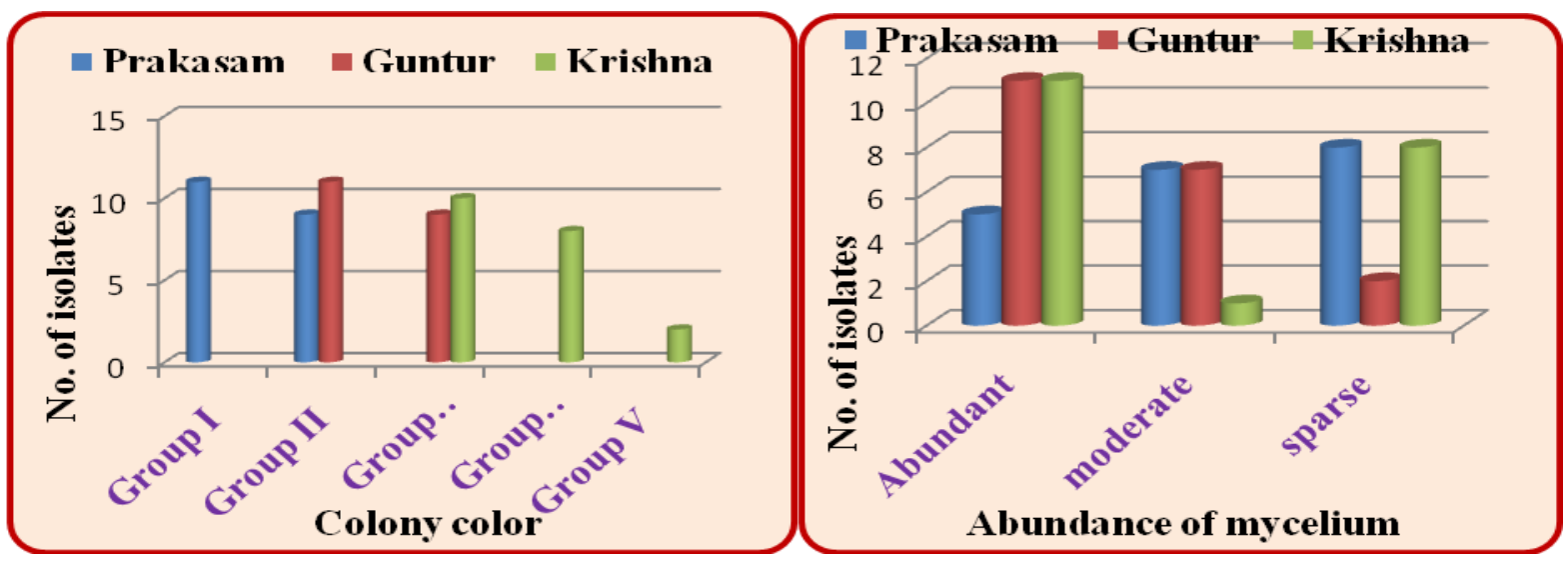




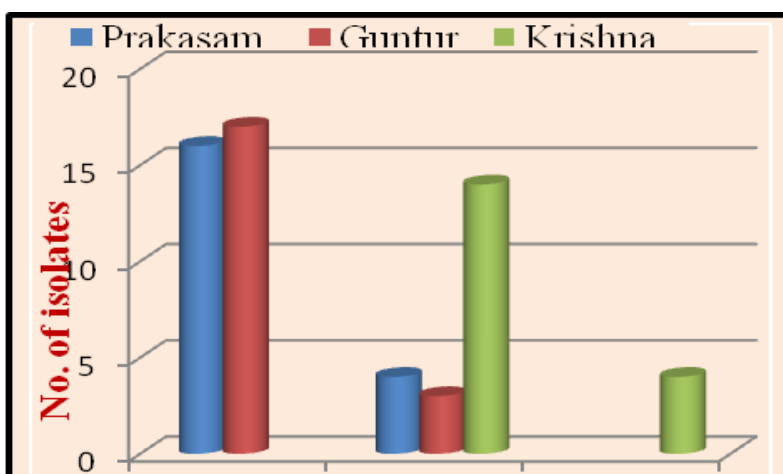

Group \$cleffintcbionitroup III

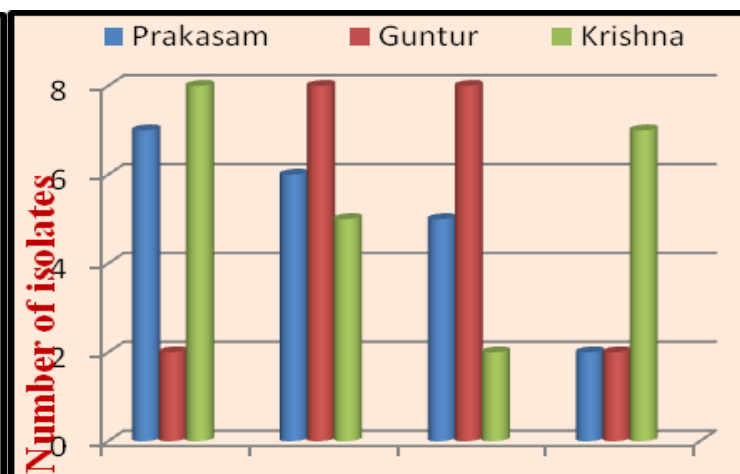

Group IGroup IGroup IIGroup IV Pattern of sclerotia

Table.1 Cultural characteristics of different isolates of Rhizoctonia solani f.sp. sasakii collected from Prakasam, Guntur and Krishna districts of Andhra Pradesh

\begin{tabular}{|c|l|l|l|c|l|l|c|}
\hline Isolate & $\begin{array}{l}\text { Hyphal } \\
\text { width } \\
\boldsymbol{\mu} \mathbf{\mu m})\end{array}$ & $\begin{array}{l}\text { Number } \\
\text { of nuclei }\end{array}$ & $\begin{array}{c}\text { Colour of } \\
\text { the } \\
\text { colony }\end{array}$ & $\begin{array}{c}\text { Colony } \\
\text { diameter } \\
\text { after } \\
\mathbf{7 2 h}(\mathbf{m m})\end{array}$ & $\begin{array}{l}\text { Growth } \\
\text { rate* }\end{array}$ & $\begin{array}{l}\text { Growth } \\
\text { pattern }\end{array}$ & $\begin{array}{c}\text { Time taken } \\
\text { to initiate } \\
\text { Sclerotia } \\
\text { (days) }\end{array}$ \\
\hline RS 1 & 5.35 & 6 & Pale brown & 66 & Fast & Moderate & 4 \\
\hline RS 2 & 5.40 & 5 & Pale brown & 57 & Fast & Moderate & 4 \\
\hline RS 3 & 6.05 & 5 & White & 45 & moderate & Abundant & 4 \\
\hline RS 4 & 5.83 & 5 & White & 69 & Fast & Moderate & 5 \\
\hline RS 5 & 7.05 & 6 & White & 44 & moderate & Slight & 5 \\
\hline RS 6 & 7.27 & 6 & White & 29 & slow & Slight & 6 \\
\hline RS 7 & 6.45 & 6 & Pale brown & 75 & Fast & Moderate & 4 \\
\hline RS 8 & 5.95 & 5 & Pale brown & 78 & Fast & Abundant & 4 \\
\hline RS 9 & 6.10 & 5 & White & 42 & Moderate & Slight & 5 \\
\hline RS 10 & 5.12 & 6 & White & 30 & Slow & Slight & 6 \\
\hline RS 11 & 5.37 & 6 & White & 45 & Moderate & Slight & 5 \\
\hline RS 12 & 5.55 & 5 & White & 44 & Moderate & Slight & 5 \\
\hline RS 13 & 6.65 & 6 & Pale & 45 & Moderate & Abundan & 5 \\
\hline RS 14 & 7.23 & 6 & Pale brown & 22 & slow & Moderate & 7 \\
\hline RS 15 & 7.98 & 6 & Pale brown & 29 & slow & Slight & 7 \\
\hline RS 16 & 6.45 & 6 & White & 64 & Fast & Abundant & 4 \\
\hline RS 17 & 5.08 & 5 & Pale brown & 40 & Moderate & Abundant & 5 \\
\hline RS 18 & 5.11 & 5 & White & 39 & Moderate & Moderate & 5 \\
\hline RS 19 & 5.86 & 5 & White & 28 & slow & Slight & 6 \\
\hline RS 20 & 6.65 & 7 & Pale brown & 39 & Moderate & Moderate & 5 \\
\hline RS 21 & 5.84 & 7 & $\begin{array}{l}\text { Yellowish } \\
\text { white }\end{array}$ & 65 & Fast & Abundant & 4 \\
\hline RS 22 & 5.48 & 6 & Pale brown & 69 & Fast & Abundant & 4 \\
\hline RS 23 & 6.44 & 7 & Pale brown & 56 & Fast & Abundant & 4 \\
\hline
\end{tabular}




\begin{tabular}{|c|c|c|c|c|c|c|c|}
\hline RS 24 & 5.23 & 6 & Pale brown & 41 & Moderate & Abundant & 4 \\
\hline RS 25 & 6.15 & 7 & Pale brown & 66 & Fast & Moderate & 5 \\
\hline RS 26 & 6.87 & 7 & $\begin{array}{l}\text { Yellowish } \\
\text { white }\end{array}$ & 69 & Fast & Abundant & 4 \\
\hline RS 27 & 5.21 & 7 & $\begin{array}{l}\text { Yellowish } \\
\text { white }\end{array}$ & 70 & Fast & Abundant & 4 \\
\hline RS 28 & 5.65 & 6 & $\begin{array}{l}\text { Yellowish } \\
\text { white }\end{array}$ & 66 & Fast & Abundant & 4 \\
\hline RS 29 & 6.51 & 6 & Pale brown & 62 & Fast & Abundant & 4 \\
\hline RS 30 & 7.57 & 6 & Pale brown & 60 & Fast & Moderate & 4 \\
\hline RS 31 & 7.14 & 5 & Pale brown & 44 & Moderate & Moderate & 4 \\
\hline RS 32 & 6.16 & 5 & $\begin{array}{l}\text { Yellowish } \\
\text { white }\end{array}$ & 51 & Fast & Moderate & 4 \\
\hline RS 33 & 5.20 & 5 & Pale brown & 55 & Fast & Moderate & 4 \\
\hline RS 34 & 6.80 & 6 & $\begin{array}{l}\text { Yellowish } \\
\text { white }\end{array}$ & 26 & slow & Abundant & 5 \\
\hline RS 35 & 7.12 & 6 & $\begin{array}{l}\text { Yellowish } \\
\text { white }\end{array}$ & 61 & Fast & Abundant & 4 \\
\hline RS 36 & 7.05 & 6 & Pale brown & 72 & Fast & Slight & 6 \\
\hline RS 37 & 6.55 & 7 & Pale brown & 29 & Slow & Slight & 6 \\
\hline RS 38 & 5.33 & 5 & $\begin{array}{l}\text { Yellowish } \\
\text { white }\end{array}$ & 46 & Fast & Moderate & 4 \\
\hline RS 39 & 5.84 & 6 & Pale brown & 55 & Fast & Abundant & 4 \\
\hline RS 40 & 6.81 & 6 & $\begin{array}{l}\text { Yellowish } \\
\text { white }\end{array}$ & 41 & Moderate & Moderate & 5 \\
\hline RS 41 & 6.25 & 6 & Brown & 32 & Moderate & Slight & 5 \\
\hline RS 42 & 6.13 & 6 & Dark brown & 34 & Moderate & Slight & 5 \\
\hline RS 43 & 5.68 & 6 & Brown & 30 & Moderate & Slight & 5 \\
\hline RS 44 & 5.26 & 6 & Pale brown & 31 & Moderate & Slight & 6 \\
\hline RS 45 & 5.11 & 5 & Dark brown & 40 & Fast & Abundant & 5 \\
\hline RS 46 & 6.60 & 5 & Dark brown & 42 & Fast & Abundant & 5 \\
\hline RS 47 & 6.47 & 6 & Dark brown & 44 & Fast & Abundant & 6 \\
\hline RS 48 & 6.80 & 6 & Pale brown & 44 & Fast & Abundant & 6 \\
\hline RS 49 & 7.55 & 6 & Brown & 45 & Fast & Abundant & 6 \\
\hline RS 50 & 5.45 & 6 & Dark brown & 50 & Fast & Abundant & 6 \\
\hline RS 51 & 5.83 & 6 & Dark brown & 54 & Fast & Abundant & 6 \\
\hline RS 52 & 5.05 & 6 & Brown & 52 & Fast & Abundant & 6 \\
\hline RS 53 & 6.25 & 6 & Brown & 36 & Moderate & Abundant & 8 \\
\hline RS 54 & 7.30 & 6 & Dark brown & 32 & Slow & Moderate & 10 \\
\hline RS 55 & 7.87 & 6 & Brown & 38 & Moderate & Abundant & 8 \\
\hline RS 56 & 6.48 & 5.5 & Brown & 39 & Moderate & Abundant & 8 \\
\hline RS 57 & 6.05 & 6 & Brown & 32 & Moderate & Slight & 8 \\
\hline
\end{tabular}




\begin{tabular}{|c|c|c|l|c|l|l|c|}
\hline RS 58 & 7.20 & 6 & Brown & 22 & Slow & Slight & No sclerotia \\
\hline RS 59 & 7.83 & 5.5 & Dark brown & 29 & Slow & Slight & No sclerotia \\
\hline RS 60 & 5.65 & 6.5 & Brown & 35 & Moderate & Slight & 8 \\
\hline RS61 & 7.44 & 6 & White & 49 & Fast & Slight & 6 \\
\hline S. Em \pm & 0.056 & 0.266 & & & & & \\
\hline $\begin{array}{c}\text { CD } \\
(0.05 \%)\end{array}$ & 0.157 & 0.737 & & & & & \\
\hline
\end{tabular}

*Scale

Isolates showing more than $40 \mathrm{~mm}$ growth after $72 \mathrm{~h}$ (fast growers)

Isolates showing more than $35-40 \mathrm{~mm}$ growth after $72 \mathrm{~h}$ (moderate growers)

Isolates showing more than $30-35 \mathrm{~mm}$ growth after $72 \mathrm{~h}$ (slow growers)

Table.2 Sclerotial characteristics of different isolates of Rhizoctonia solani f. sp. sasakii

\begin{tabular}{|c|c|c|c|c|c|c|c|}
\hline Isolate & $\begin{array}{l}\text { Colour } \\
\text { of sclerotia }\end{array}$ & $\begin{array}{l}\text { Number of } \\
\text { sclerotia/ } \\
\text { petri dish }\end{array}$ & $\begin{array}{l}\text { Manner of } \\
\text { sclerotial } \\
\text { formation }\end{array}$ & $\begin{array}{l}\text { Location of } \\
\text { sclerotia }\end{array}$ & $\begin{array}{l}\text { Clump } \\
\text { formation }\end{array}$ & Size & $\begin{array}{l}\text { Texture of } \\
\text { sclerotia }\end{array}$ \\
\hline RS 1 & Light brown & Very good & Centre & Aerial surface & Moderate & Large & Rough \\
\hline RS 2 & Dark brown & Very good & Peripheral & Aerial surface & Moderate & Large & Rough \\
\hline RS 3 & Light brown & Very good & Centre & Aerial surface & Moderate & Large & Rough \\
\hline RS 4 & Light brown & Good & Centre & Aerial surface & Moderate & Large & Rough \\
\hline RS 5 & Light brown & Good & Centre & Aerial surface & Moderate & Micro & Smooth \\
\hline RS 6 & Light brown & Excellent & Peripheral & Embeded & Less & Small & Smooth \\
\hline RS 7 & Light brown & Very good & $\begin{array}{l}\text { Peripheral } \\
\text { \&centre }\end{array}$ & Aerial surface & Less & Small & Smooth \\
\hline RS 8 & Light brown & Very good & Scatter & Aerial surface & Less & Large & Rough \\
\hline RS 9 & Light Brown & Good & $\begin{array}{l}\text { Peripheral } \\
\text { \&centre }\end{array}$ & Aerial surface & Moderate & Large & Rough \\
\hline RS 10 & Dark brown & Very good & Peripheral & Embeded & Moderate & Large & Rough \\
\hline RS 11 & Light brown & Very good & $\begin{array}{l}\text { Peripheral } \\
\text { \&centre }\end{array}$ & Aerial surface & Less & Large & Rough \\
\hline RS 12 & Light brown & Good & $\begin{array}{l}\text { Peripheral } \\
\text { \&centre }\end{array}$ & Embeded & Moderate & Large & Rough \\
\hline RS 13 & Light brown & Good & Centre & Aerial surface & Moderate & Small & Smooth \\
\hline RS 14 & Light brown & Good & Centre & Aerial surface & Less & Large & Rough \\
\hline RS 15 & Light brown & Good & Peripheral & Aerial surface & Less & Large & Rough \\
\hline RS 16 & Light brown & Very good & $\begin{array}{l}\text { Peripheral } \\
\text { \&centre }\end{array}$ & Aerial surface & Less & Large & Rough \\
\hline RS 17 & Dark brown & Very good & Scatter & Aerial surface & Less & Large & Rough \\
\hline RS 18 & Light brown & Very good & Centre & Aerial surface & Moderate & Large & Rough \\
\hline RS 19 & Light brown & Very good & Peripheral & Embeded & Less & Large & Rough \\
\hline RS 20 & Dark brown & Good & Peripheral & Aerial surface & Less & Small & Smooth \\
\hline RS 21 & Light brown & Very good & Peripheral & Aerial surface & Moderate & Large & Rough \\
\hline RS 22 & Light brown & Very good & Peripheral & Aerial surface & Moderate & Large & Rough \\
\hline RS 23 & Light brown & Very good & Peripheral & Aerial surface & Moderate & Large & Rough \\
\hline RS 24 & Light brown & Very good & Peripheral & Aerial surface & Moderate & Large & Rough \\
\hline RS 25 & Light brown & Good & Peripheral & Aerial surface & More & Large & Rough \\
\hline RS 26 & Light brown & Very good & $\begin{array}{l}\text { Peripheral } \\
\text { \&centre }\end{array}$ & Aerial surface & Moderate & Large & Rough \\
\hline RS 27 & Light brown & Excellent & Peripheral & Aerial surface & Moderate & Large & Rough \\
\hline RS 28 & Light brown & Excellent & Peripheral & Aerial surface & Moderate & Large & Rough \\
\hline
\end{tabular}




\begin{tabular}{|c|c|c|c|c|c|c|c|}
\hline & & & \&centre & & & & \\
\hline RS 29 & Light brown & Very good & $\begin{array}{l}\text { Peripheral } \\
\& \text { centre }\end{array}$ & Aerial surface & Moderate & Large & Rough \\
\hline RS 30 & Light brown & Very good & $\begin{array}{l}\text { Peripheral } \\
\& \text { centre }\end{array}$ & Aerial surface & More & Large & Rough \\
\hline RS 31 & Light brown & Good & $\begin{array}{l}\text { Peripheral } \\
\& \text { centre }\end{array}$ & Aerial surface & Less & Large & Rough \\
\hline RS 32 & Light brown & Good & Peripheral & Aerial surface & Less & Large & Rough \\
\hline RS 33 & Light brown & Very good & Scatter & Aerial surface & Less & Large & Rough \\
\hline RS 34 & Light brown & Excellent & Peripheral & Aerial surface & Less & Large & Rough \\
\hline RS 35 & Light brown & Very good & Peripheral & Aerial surface & More & Large & Rough \\
\hline RS 36 & Light brown & Good & $\begin{array}{l}\text { Peripheral } \\
\& \text { centre }\end{array}$ & Aerial surface & Moderate & Large & Rough \\
\hline RS 37 & Light brown & Good & $\begin{array}{l}\text { Peripheral } \\
\text { \&centre }\end{array}$ & Aerial surface & More & Large & Rough \\
\hline RS 38 & Dark brown & Good & $\begin{array}{l}\text { Peripheral } \\
\& \text { centre }\end{array}$ & Aerial surface & Moderate & Large & Rough \\
\hline RS 39 & Dark brown & Good & $\begin{array}{l}\text { Peripheral } \\
\& \text { centre }\end{array}$ & Aerial surface & Moderate & Large & Rough \\
\hline RS 40 & Dark brown & Good & Scatter & Aerial surface & Moderate & Large & Rough \\
\hline RS 41 & Dark brown & Good & Centre & Aerial surface & More & Large & Rough \\
\hline RS 42 & Dark brown & Good & Peripheral & Embeded & More & Large & Rough \\
\hline RS 43 & Dark brown & Good & Centre & Embeded & More & Large & Rough \\
\hline RS 44 & Dark brown & Good & Centre & Embeded & Moderate & Large & Rough \\
\hline RS 45 & Dark brown & Good & Centre & Aerial surface & Moderate & Large & Rough \\
\hline RS 46 & Dark brown & Good & Scatter & Embeded & More & Large & Rough \\
\hline RS 47 & Dark brown & Very good & Scatter & Aerial surface & More & Large & Rough \\
\hline RS 48 & Deep dark brown & Very good & Scatter & Aerial surface & More & Large & Rough \\
\hline RS 49 & Dark brown & Very good & $\begin{array}{l}\text { Peripheral } \\
\text { \&centre }\end{array}$ & Aerial surface & More & Large & Rough \\
\hline RS 50 & Deep dark brown & Very good & Centre & Embeded & Moderate & Large & Rough \\
\hline RS 51 & Deep dark brown & Very good & $\begin{array}{l}\text { Peripheral } \\
\& \text { centre }\end{array}$ & Embeded & Moderate & Large & Rough \\
\hline RS 52 & Dark brown & Very good & Scatter & Embeded & More & Large & Rough \\
\hline RS 53 & Deep dark brown & Good & Scatter & Aerial surface & Moderate & Large & Rough \\
\hline RS 54 & Dark brown & Good & Centre & Embeded & Moderate & Large & Rough \\
\hline RS 55 & Dark brown & Good & Centre & Embeded & Moderate & Large & Rough \\
\hline RS 56 & Dark brown & Good & Centre & Embeded & Moderate & Large & Rough \\
\hline RS 57 & Dark brown & Good & Peripheral & Embeded & More & Large & Rough \\
\hline RS 58 & \multicolumn{3}{|c|}{ No sclerotia formation } & & & & \\
\hline RS 59 & \multicolumn{3}{|c|}{ No sclerotia formation } & & & & \\
\hline RS 60 & Dark brown & Good & Scatter & Aerial surface & More & Large & Rough \\
\hline RS 61 & Brown & Good & Peripheral & Aerial surface & More & Large & Rough \\
\hline
\end{tabular}

\section{Time taken for sclerotia formation}

All the isolates under the study showed great variation in the time taken for initiation of sclerotia formation, which ranged from 4- 10 days (Table 1).

Twenty one isolates took 4 days for initiation,
17 isolates took 5 days, 12 isolates took 6 days and 2 isolates took 7days, 5 isolates in 8 days and 1 isolate took 10 days for sclerotia formation. Sclerotial formation was not observed in RS 58 and RS 59 isolates.

\section{Sclerotial variability of $\boldsymbol{R}$. solaniisolates}


Observations on the variation in the sclerotial characteristics such as the colour of the sclerotia, location of formation, pattern of production, sclerotia number were recorded after 15 days of inoculation.

\section{Sclerotia colour}

The mycelia of most of the isolates formed small spherical sclerotia that were initially cream coloured then light yellowish brown and eventually turned to strong brown or dark reddish brown colour. Based on the colour of the sclerotia, they were categorised into three groups,category I- light brown, category IIdark brown and category III- deep dark brown (Table 2). Colour of the sclerotia was observed using Munsell's soil colour chart (1954).

The sclerotia produced by different isolates in the present study were initially white and turned brown with maturity. Among the 60 isolates, 33 isolates produced pale brown sclerotia where as 21 isolates produced dark brown sclerotia and the remaining 6 isolates recorded deep dark brown sclerotia. Prakasam and Guntur district isolates produced pale brown and dark brown sclerotia where as Krishna district isolates produced dark brown and deep dark brown sclerotia. Further it was also observed that two isolates RS 58 and RS 59 did not produce any sclerotia.

\section{Pattern of sclerotial formation}

The isolates were divided into 4 categories i.e., in the I category, the sclerotia were produced more towards the centre of the colony, in II category sclerotia were produced more or less in circular manner concentrated towards periphery, in III category, the sclerotia were irregular, sparse and concentrated both in centre and periphery, in IV category, sclerotia were formed in irregular manner scattered all over the colony surface. Among the 60 isolates, 17 isolates produced sclerotia in category I, 15 isolates in category II, 15 in category III and 11 in category IV.

\section{Location of sclerotial formation}

On the basis of the location of sclerotial formation in the fungal colony, the isolates were categorized into two groups. The first group included those isolates where sclerotiawere formed in the aerial surface and the second group included the isolates where sclerotia were embedded in the fungal mycelium (Plate 4.5). Out of the 60 isolates, 45 isolates produced sclerotia in group I category whereas the remaining 13 isolates were grouped under group II with sclerotia embedded in the mycelium.

\section{Clump formation}

Sclerotia were aggregated in all the isolates and formed clumps. Based on the clump formation the isolates were categorized in to 3 groups. Group I included more clump formation, Group II included moderate clump formation whereas the group III less clump formation. Among the 60 isolates, 16 isolates were assigned in group I (more clump), 30 isolates were included in group II (moderate clump) whereas 14 isolates were included under group III (less clump) (Plate 4.3).

\section{Number of Sclerotia}

The number of sclerotia was recorded 15 days after incubation and the isolates were categoried into three groups as, good, very good and excellent on the basis of the number of sclerotia produced. Sclerotia forming isolates were categorized as poor, 1-40 sclerotia good, 41-60 very good and above 60 excellent. Accordingly majority of the $R$. solani isolates belonged to good (50per cent isolates), and very good (40 per cent isolates) and only 6.6 per cent isolates produced 
excellent number of sclerotia and 2 per cent of isolates did not produce any sclerotia.

\section{Size of sclerotia}

In the present study size of sclerotia of $R$. solani f.sp. sasakii was categorized into 2 groups, group I large and group II small based on diameter of sclerotia, 1-1.5 mm small and above $1.5 \mathrm{~mm}$ large. Out of the 60 isolates, 53 isolates produced large sclerotia and 5 isolates produced small sclerotia and 2 isolates produced no sclerotia.Out of the 20 isolates collected from Prakasam district 15 isolates produced large sclerotia and 5 isolates produced small sclerotia. All the isolates of Guntur district produced large sclerotia. Among the 20 isolates from Krishna District 18 isolates produced large sclerotia and two isolates produced no sclerotia.

\section{Texture of sclerotia}

On the basis of texture of sclerotia, $R$. solani isolates were classified into two groups i.e, smooth and rough. The smooth type of sclerotia was formed only in 8.3 per cent isolates (five) in the culture media and rough type by 88.3 per cent (53) isolates and the remaining 3.3 per cent (2) isolates produced no sclerotia.

The above results are in agreement with the reports of earlier researchers. Anderson (1982) reported that colour was the only character that clearly distinguished the sclerotia. Srinivas (2002) assigned the sclerotia of $R$. solani isolates causing BLSB in maize into three different colour groups based on pigmentation. Similar categorisation of the sclerotia produced by the $R$. solani isolates in the rice was assigned as per Munsell's soil colour chart by Amita Singh et al., (1999), Thind and Aggarwal (2008) in potato and rice isolates. The arrangement and pattern of formation of sclerotia produced by
$R$. solaniisolates in the culture varied greatly and were divided into four categories. However most of the isolates had sclerotia which conglomerated at the centre of the colony. Such type of categorisation based on the pattern of formation and arrangement among maize isolates of $R$. solaniwas done by Srinivas (2002), rice isolates by Amita Singh et al., (1999) and sclerotia from potato and rice isolates by Thind and Aggarwal (2008).

In the present investigation isolates RS 58 and Rs 59 did not produce sclerotia. Sclerotia may be absent in some R.solani isolates under certain cultural conditions therefore the absence of sclerotia does not exclude a mycelium from $R$. solani. Similar findings reported by Meyer (1965) and Parmeter and Whitney (1970).

Higher sclerotial aggregation was observed in 16 isolates. The behaviour of the pathogen to grow quickly and produce sclerotia may increase the chances of pathogen to survive in the next season. High sclerotial aggregation, may hinder the quick dispersal of sclerotia and increases the chances of germination whenever they are present. In most of the isolates, (71.6 per cent of isolates) sclerotia were produced on the aerial surface of the colony, while 25 per cent of isolate had embedded sclerotia. Akhtar et al., (2009) revealed that the sclerotia of maize $R$. solani isolates $l t$ and $R f$ were located on the surface of the colony; isolate $H c$ produced on subsurface while in isolates $B e$ and $J r$, sclerotia were embedded in the medium. Zhang et al., (1995) observed variation based on sclerotiacolour, shape or formation patterns AG-1 IA, Ag-2-2 IIIB cool- season turf grasses and AG-4: assigned the isolates of the $R$. solani tentatively to an AG based on sclerotial characteristics

The results with respect to morphological, cultural and sclerotial characters of $R$. solani 
observed in the present investigations have also been recorded and described by several workers (Duggar, 1915; Reyes, 1941; Singh and Sharma, 1976; Maiti, 1978, Srinivas, 2002, Sharma et al., 2004 and Akhtar et al., 2009 in maize and other crops).

\section{References}

Agrios, G. N. 2005. Plant Pathology Fifth edition., Elsevier Academic Press, New York. 901.

Akhtar, J., Kumar Jha, V and Lal, H.C. 2009. Occurrence of banded leaf and sheath blight of Maize in Jharkand with reference to diversity in Rhizoctonia solani.Asian Journal of Agricultural Sciences.1 (2): 32-35.

Amita Singh, Singh, U.S., Willocquet, L and Savary, S. 1999. Relationship among cultural/ morphological characteristics, anastomosis behaviour and pathogenicity of Rhizoctonia solani Kuhn on rice. Journal of Mycology and Plant Pathology.29 (3):306-316.

Basu, A., Podder, M., Prasanta, K and Sengupta. 2004. Variability and anastamosis among the rice isolates of Rhizoctonia solani. Indian Phytopathology.57(1):70-72.

Burpee, L. L., Sanders, P. L., Cole, H. Jr and Sherwood, R.T. 1980. Anastomosis groups among isolates of Ceratobasidium cornigerum and related fungi. Mycologia.72:689-701.

Duggar, B. M. 1915. Rhizoctonia crocorrum (Pers.) Dc and $R$. solani Kühn (Corticium vagum B. \& C.) with notes on other species. Annals of Missouri Botanical Garden. 2:403-458.

Guleria, S., Aggarwal, R and Thind, T. S. 2007. Morphological and pathological variability in rice isolates of Rhizoctonia solani and molecular analysis of their genetic variability. Journal of Phytopathology.155 (11-12):
654-661.

Khodayari, M, Safaie, N and Shamsbakhsh, M. 2009. Genetic diversity of Iranian AG1-IA isolates of Rhizoctonia solani, the cause of rice sheath blight, using morphological and molecular markers. Journal of Phytopathology.157:708714.

Maiti, S. 1978. Two new ear rots of maize from India. Plant Disease Report.62:1074-1076.

Matsumoto, $\mathrm{T}$ and Yamoto, W. 1935.HypochnussasakiiShirai in comparison with Corticium stevensii Burt. And Corticium coleroga (Cook) V. Hohn. Trans. Nat. Hist. Soc., (Formosa). 25:161-175.

Matsumoto, T. 1921. Studies on the physiology of the fungi XII. Physiological specialization in Rhizoctonia solani Kuhn. Annals of Missouri Botanical Garden. 8:1-62.

Matz, J. 1921. The Rhizoctonias of Porto Rico. Porto Rico Department of Agriculture Journal.5:1-31.

Meena, B., Ramamoorthy, V and Muthusamy, M. 2001. Morphological and pathological variations in isolates of Rhizoctonia solani causing sheath blight of rice. Plant Disease Research.16 (2):166-172.

Meena, R. L., Rathore, R. S and Mathur, K. 2003. Efficacy of Biocontrol agents against Rhizoctonia solani f. sp. sasakii causing banded leaf and sheath blight of maize. Journal of Mycology and Pathology.33:310 - 312.

Meyer, R.W. 1965. Heterokaryosis and nuclear phenomenon in Rhizoctonia. Ph.D. Thesis, University of California, Berkeley, 118.

Munsell's Soil Colour Chart. 1954. Munsell Colour Co. Inc. Baltimore, Maryland, USA.

OuS.H., 1985. Rice Diseases.2nd ed. Commonwealth Mycological Institute, 
Kew, UK, 380.

Parmeter, J. R., Jr. and Whitney, H.S. 1970. Taxonomy and nomenclature of the imperfect state.(In:) J.R. Parameter Jr., (Ed.) Biology and Pathology of Rhizoctonia solani.7-19. University of California Press, Berkeley. 255.

Reyes, G.M.1941. Notes on diseases affecting maize in Philippines. Phillippines Journal of Agriculture.12:61-69.

Sharma, R. R., Gour, H. N and Rathore, R.S. 2004. Etiology of banded leaf and sheath blight symptoms on maize. Journal of Mycology and Plant Pathology.34 (1): 56-59.

Singh, B. M and Sharma, Y. R.1976. Evaluation of maize germplasm to banded sclerotial disease and assessment of yield loss. Indian Phytopathology. 29(2): 129-132.

Singh, V., Singh, U. S., Singh, K. P., Singh, $\mathrm{M}$ and Kumar, A. 2002. Genetic diversity of Rhizoctonia solani isolated from rice differentiation by morphological characteristics, pathogenicity, anastomosis behaviour and RAPD finger printing. Journal of Mycology and Plant Pathology.32:332344.

Sneh, B., Burpee, L and Ogoshi, A. 1991. Identification of Rhizoctonia Species. St Paul, MN, APS Press.133.

Srinivas, P. 2002. Studies on genetic and pathogenic variation in $R$. solanif. $s p$. sasakiiincitant of banded leaf and sheath blight of maize. Ph.D. thesis, Division of Plant Pathology, IARIIndia.155.

Sweetingham, M. W and MacNish, G. C. 1994.Rhizoctonia isolation, identification and pathogenicity. $A$ Laboratory Manual. Perth, WA, Australia, Department of Agriculture, Misc Publications 1492. 30.

Thind, T. S and Rohit Aggarwal. 2008. Characterization and pathogenic relationships of Rhizoctonia solani isolates in a potato-rice system and their sensitivity to fungicides. Journal of Phytopathology.156 (10): 615-621.

Thomas, K.S. 1925. Onderzockinger over Rhizoctonia Electr. Drukkerij "De Industries J. Van Drutten Utrecht. 98.

Toda, T., Hyakumachi, M., Arora, D. K. 1999.Genetic relatedness among and within different Rhizoctonia solani anastomosis groups as assessed by RAPD, ERIC and REP-PCR. Microbioogical Research. 154:247-258.

$\mathrm{Tu}, \quad$ C.C and Kimbrough, J.W.1978. Systematics and phylogeny of fungi in the Rhizoctonia complex. Botanical Gazette.139: 454-466.

Zhang, P.Q., De Leon, C and Yang, II.1995. Investigation on the yield loss caused by Rhizoctonia solani on maize. Report of Gaungxi Maize Research Institute (Unpublished)

\section{How to cite this article:}

Bindu Madhavi Gopireddy, G. Uma Devi, K.Vijayakrishna Kumar, T. Ramesh Babu and Naidu, T. C. M. 2017. Cultural and Morphological Characterization of Rhizoctonia solani f. sp. sasakii Isolates Collected from Different Districts of Andhra Pradesh. Int.J.Curr.Microbiol.App.Sci. 6(11): 3457-3469. doi: https://doi.org/10.20546/ijcmas.2017.611.407 